\title{
Avoidance Behavior in Adults with Developmental Coordination Disorder is Related to Quality of Life
}

\author{
John Joe Forde ${ }^{1} \cdot$ Sinéad Smyth ${ }^{1}[$
}

Accepted: 9 August 2021 / Published online: 24 September 2021

(c) The Author(s) 2021

\begin{abstract}
Although it is now widely accepted that developmental co-ordination disorder (DCD) persists into adolescence and adulthood, few studies have assessed the concept of Quality of Life (QOL) within the adult DCD population. This is despite a growing recognition that children and adolescents with DCD experience poorer QOL than their typically developed peers. The small body of literature which has examined QOL in adults with DCD has reported that they also report lower levels of QOL as a result of motor coordination deficits. Little research has investigated the factors that result in adults with DCD reporting lower QOL than their typically developed counterparts. The current study explored the relationships between QOL and motor coordination, and QOL and three individual subtypes of avoidance within an adult DCD sample. Eighty-five adults (27 males; 58 females) who reported a diagnosis of, or believe that they have DCD, completed an online questionnaire that consisted of three already established questionnaires in order to measure the concepts of QOL, motor coordination and avoidance, respectively. A hierarchal multiple regression revealed that all three types of avoidance behaviors were significant predictors of QOL scores, while levels of motor coordination did not significantly predict scores of QOL. These results highlight the need for clinicians to develop interventions to help reduce avoidance behavior and social impairment, in order to improve QOL within the DCD adult population.
\end{abstract}

Keywords DCD · Adults · Dyspraxia $\cdot$ Clumsy $\cdot$ Neurodevelopmental $\cdot$ Avoidance Quality of life

\section{Introduction}

Despite being one of the most common neuro-developmental disorders amongst children, it is still regarded as being very much under-recognised (Missiuna et al., 2006). This can be partially explained by the relatively common co-occurrence of

Sinéad Smyth

sinead.smyth@dcu.ie

1 School of Psychology, Dublin City University, Dublin, Ireland 
DCD with other disorders, with attention deficit hyperactive disorder (ADHD), learning disabilities and autistic spectrum disorders (ASD) being the most common co-existing neuro-developmental disorders (Blank et al., 2012). While it was once thought that DCD could be outgrown with age, longitudinal research now suggests that abnormal difficulties in gross and/or fine motor coordination and problems with learning new motor skills may persist through adolescence (Cantell et al., 1994) although these may present different for males relative to females (Cleaton et al., 2021). Such difficulties persist for almost half of all children diagnosed with DCD (Cantell et al., 1994; Geuze \& Börger, 1993) and then through adulthood for those that are most severely affected (Cousins \& Smyth, 2003).

There is a growing body of literature suggesting that the broad range of heterogeneous difficulties experienced by children and adolescents with DCD has negative emotional, behavioral, social and academic repercussions (Cantell et al., 1994; Dewey et al., 2002; Hill et al., 2011; Smyth and Anderson, 2000; Tal-Saban, et al., 2014a). For instance, while in school, children with DCD have been found to spend more time alone (Smyth \& Anderson, 2000), be socially immature (Gillberg \& Gillberg, 1989), have lower levels of self-esteem and self-worth (Piek et al., 2000; Shaw et al., 1982), and are more introverted and anxious than their neurotypical peers (Schoemaker \& Kalverboer, 1994). Studies have also demonstrated that children with DCD have poor concentration levels (Losse et al., 1991) and poorer academic achievement (Cantell et al., 1994) relative to their peers.

Although there has been extensive research conducted assessing the difficulties faced on a daily basis by individuals with DCD within the children and adolescent populations in recent years, the developmental course and outcomes of DCD into adulthood remains relatively unexplored (Barnett et al., 2011). Findings from studies assessing the symptoms and implications of DCD within the adolescent and adult DCD populations suggests that many of the deficits and difficulties experienced in childhood DCD, such as poor motor coordination and organisational skills, still remain (Tal-Saban et al., 2014a). Deficits in gross- and fine-motor skills (Cantell et al., 2003), handwriting (Barnett et al., 2011; Kirby et al., 2008) and time management skills (Tal-Saban et al., 2014b) are the most prominent characteristics of DCD within the adolescent and adult population. Research suggests that such difficulties can impair individuals in many aspects of everyday life such as; organisational skills, driving (de Oliveira \& Wann, 2011; Kirby et al., 2011a) and retaining employment status (Kirby et al., 2013).

The concept of participation, defined as a person's involvement in daily activities in a variety of environments, roles and life situations (Liberman et al., 2013), has been explored quite extensively within the DCD population. Research regarding participation amongst individuals with chronic conditions suggests that the severity of their physical limitations is one of the primary determinants of impaired participation (van Campen \& Iedema, 2007). Studies assessing levels of participation exhibited by individuals with DCD have yielded evidence suggesting that children with DCD engage in significantly less participation behavior than their neurotypical peers (Sylvestre et al., 2013; Mandich et al., 2003; Rosenblum \& Engel-Yeger, 2014). Similar studies have also been replicated within the adult population, with Kirby et al. (2008) suggesting that adults with DCD participate significantly less in 
physical activities and are more likely to live at home with their parents. The authors suggest that this is due to continued need for support in daily activities. Cousins and Smyth (2003) highlighted that many adults with DCD are unable or unwilling to learn how to drive as a result of poor motor coordination or skill-learning difficulties. This results in their levels of independence and social and employment opportunities being reduced substantially.

Ottenbreit and Dobson (2004) defined avoidance behavior as the act of refraining from, or escaping from, an action, person or thing. Avoidance behavior is a coping style utilised by many individuals who are living with disabilities and/or chronic illness (Dettling-Ihnenfeldt et al., 2016). The maintenance of avoidance behaviors can be explained through the principles of learning because these behaviors are negatively reinforced through the avoidance of aversive situations (Zeiss et al., 1979). However, these behaviors can result in the engagement in depressive like symptoms, such as an increase in passive behavior (e.g., withdrawal and isolation) and increased rate of escaping and avoiding potentially aversive conditions (Petts et al., 2016). One well-established measurement of avoidance behavior is the Behavioral Activation Depression Scale (BADS; Kanter et al., 2007). The developers of the BADS (Kanter et al., 2007) suggest that levels of avoidance behaviors can be predictive of future depressive tendencies.

As aforementioned, a small body of literature exists that has assessed participation in daily tasks and social roles amongst the DCD population. However, little research has specifically assessed the distinct concept of avoidance and the types of avoidance behaviors individuals with DCD engage in. Indeed, only one known study has examined this. Kirby et al. (2011b), in a study of young adults with DCD reported that adults with DCD reported social difficulties and isolation. The results yielded from this study suggested that almost three quarters of the participants regularly avoid getting involved in team games and going dancing, with participants reporting that this is largely due to their coordination problems. Research of participation in young boys with DCD has also highlighted that individuals with DCD tend to avoid opportunities for social engagement, preferring to spend leisure time alone (Poulsen et al., 2007).

Given that DCD has profound effects on many aspects of an individual's life, several studies have assessed whether the condition affects children's QOL (Flapper \& Schoemaker, 2013; Zwicker et al., 2013) and more recently, adolescents and adults (Hill et al., 2011; Tal-Saban et al., 2014a). QOL has been defined as an "individuals' perception of their position in life in the context of the culture and value systems in which they live and in relation to their goals, expectations, standards and concerns" (WHOQOL Group, 1995; p.1405). It is a subjective evaluation which is embedded in a cultural, social and environmental context (WHOQOL Group, 1995). For individuals with a disability, QOL incorporates the influence and interaction of environmental factors, the impact of disability on life and the opportunities open to the individual (Patrick et al., 2000). According to Megari (2013), most chronic illnesses, such as cancer, heart diseases, HIV or diabetes, for example, have the potential to significantly reduce an individual's QOL. Similarly, van Campen and Iedema (2007) suggest that the observed differences in QOL levels may be explained by lower participation and physical limitations among individuals living with chronic disease. 
Indeed, individuals with DCD across all age groups report significantly poorer QOL than their peers, possibly due to the academic, social, behavioral and emotional ramifications associated with DCD (Kaplan et al., 2001; Tal-Saban et al., 2012a).

The results of a qualitative study conducted by Mandich et al. (2003) suggests that the QOL of children with DCD is negatively affected as a result of motor deficits, difficulties in social participation and activities of daily living. Studies of adolescents and adults have yielded similar results with many researchers suggesting that motor co-ordination deficits, levels of participation and avoidance of certain social situations, among other factors, effects QOL within the DCD population (Cousins \& Smyth, 2003; Tal-Saban et al., 2014b). Although many researchers now suggest that such associations may exist, there is still very much an absence of research assessing the direct relationships between levels of motor coordination, participation, and avoidance behaviors on QOL in adults with DCD.

One such longitudinal study conducted by Tal-Saban et al. (2014a) assessed QOL and participation across three different young adult groups: DCD, borderline DCD and non-DCD groups, respectively. The results indicated that both the DCD and borderline DCD groups reported significantly lower scores of participation, QOL and life satisfaction than their typically developed peers in the nonDCD group. Although the results were consistent with those of Hill et al. (2011), the researchers highlight the need for further research exploring the implications of motor coordination difficulties on QOL and participation, in order to develop interventions that may help improve the lives of those who live with DCD. This need is further emphasised by studies suggesting that adults with DCD experience poorer mental health (Kirby et al., 2013) and are at an increased risk of developing depression and high levels of anxiety compared to their neurotypical peers (Hill \& Brown, 2013).

The current study aimed to further the current DCD literature by examining: (1) whether levels of motor coordination has a direct relationship on QOL and (2) whether levels of avoidance behaviors are directly associated with QOL in adults with DCD. As previously outlined, studies conducted by Tal-Saban et al. (2014a) and Cousins and Smyth (2003) found that when compared to a non-DCD sample, adolescents and adults with DCD report lower QOL. The current study attempted to build upon this research further and assess whether levels of motor coordination directly influence ratings on QOL. Given the absence of research specifically examining the concept of avoidance behavior, and more specifically its impact on the QOL of adults with DCD, the current study attempts to help fill this gap in the literature by assessing whether levels of engagement in avoidance behaviors directly influences QOL within this population. Firstly, we hypothesised that higher scores on three subscales of the Behavioral Activation Depression Scale (BADS; Kanter et al., 2007), School/Work Impairment, Social Impairment and Avoidance, would be associated with poorer QOL scores. Secondly, in accordance with previous literature, we hypothesised that adults with DCD who report experiencing greater levels of motor coordination difficulties on a daily basis will report poorer QOL. 


\section{Method}

\section{Participants}

A sample of 94 participants (34 males and 60 females), aged between 18 and 63 $(\mathrm{M}=32.65, \mathrm{SD}=11.77)$ were recruited through the Dyspraxia/DCD Association of Ireland and various social media platforms. Participation was restricted to individuals who were at least 18 years old and had previously been diagnosed with DCD or believed that if clinically assessed; they would receive a diagnosis of DCD. 25 (27.6\%) of the participants also reported a diagnosis or a suspected diagnosis of a comorbid disorder (eg., dyslexia, autism and type 2 diabetes). In keeping with other research in the area (Tal-Saban et al., 2012b), participants who reported a diagnosis or suspected diagnosis of a comorbid disorder were included in the study.

\section{Design}

This exploratory study was designed to examine whether levels of engagement in avoidance behavior and levels of motor coordination have a direct effect on the QOL of adults with DCD. An online survey was compiled, consisting of several demographic questions and three standardised self-report questionnaires relating to the concepts of QOL, motor coordination difficulties and avoidance behaviors, respectively (see below).

\section{Materials}

\section{Demographic Questions}

Participants were presented with several short demographic questions concerning age, gender, DCD diagnosis and comorbid disease diagnosis. Individuals who did not report a diagnosis of DCD were asked if they believed they would receive a diagnosis of DCD if clinically tested.

\section{Quality of Life Scale (QOLS; Burckhardt et al., 1989)}

Quality of Life was measured using the 16-item QOLS (Burckhardt et al., 1989). This measure assesses five conceptual domains of QOL: material and physical well-being, relationships with other people, social, community and civic activities, personal development and fulfilment, and recreation. Participants rated their present level of satisfaction with the specific factors outlined in each item using a seven-point scale, referred to as the delighted-terrible scale (Wahl et al., 2004). Item scores are totalled which results in total participant scores ranging between 16 and 112, with higher scores indicating a better QOL. Although the QOLS was originally developed through research on a healthy population, it has been validated and used successfully within populations with various chronic illnesses (Burckhardt \& 
Anderson, 2003; Wahl et al., 1998; Borge et al., 2011). Burckhardt and Anderson (2003) suggest that the average total score for healthy adults' populations is 90 . The Cronbach's alpha in the current sample was 0.836 .

\section{The Behavioral Activation Depression Scale (BADS; Kanter et al., 2007)}

For the purpose of this study, several subscales of the BADS (Kanter et al., 2007) were adopted to assess avoidance behaviors. The original BADS (Kanter et al., 2007 ) is a 25-item questionnaire, consisting of four subscales. The four subscales include; (1). "Activation", a seven-item subscale, which refers to focused, goaldirected activation and fulfilment of scheduled tasks. (2). "Avoidance", a nine-item subscale which represents avoidance of negative aversive states and engagement in rumination. (3). "Work/School Impairment", a five-item subscale which relates to the repercussions of inactivity on work and educational duties and (4) "Social Impairment", a five-item subscale which represents similar social consequences and social isolation. The three subscales adopted for the current study were Avoidance, Work/School Impairment and Social Impairment.

Within the BADS, participants were instructed to "Please read each statement carefully and then circle the number which best describes how much the statement was true for you during the past week, including today." Participants rated each item using a 7-point scale ranging from 0 (not at all) to 6 (completely). Scores within each subscale were added to give a total subscale score. Higher scores in each subscale reflect greater avoidance behavior. Previous studies have found that each individual subscale of the BADS used in this study has good internal consistency, and test-retest reliability (Kanter et al., 2007).

\section{Adolescents and Adults Coordination Questionnaire (AAC-Q; Tal-Saban et al., 2012b)}

The AAC-Q is a brief, user friendly and ecologically valid 12-item questionnaire, developed to screen for DCD amongst adolescents and adults (Tal-Saban et al., 2012 b). It is a standardised questionnaire within which respondents are asked to rate how often they experience various motor coordination difficulties as described in each individual item. Participants rate how often they encounter such difficulties on a 5-point Likert scale, ranging from never or 0\% to always or $100 \%$.

Within research, the AAC-Q is predominantly used to assign participants to one of three groups: probable DCD, suspected borderline DCD or control/non-DCD groups. Higher total scores are indicative of worse motor coordination function. Studies using the AAC-Q has provided sufficient evidence of reliability and validity for the tool in measuring motor coordination deficits and identifying suspected DCD (Tal-Saban et al., 2012a, 2012b). Although the developers of the AAC-Q recommend that for DCD research purposes, only participants who score above the probable DCD cut-off should be included for data analysis, they highlight that it is not necessary. A mean score of 19.26 on the AAC-Q was found within a large random 
sample size of 2379 of young adults aged between 19-25 (Tal-Saban et al., 2012b). In the present study, the Cronbach's alpha was 0.82 .

\section{Qualtrics}

The online survey software "Qualtrics" was used for participants to access and complete the research questionnaire. A web-link was provided in the recruitment email sent out by the DCD/Dyspraxia Association of Ireland to its members and in the recruitment advertisements on social media platforms. Participants were able to access the questionnaire on the Qualtrics software using a phone or computer with access to the internet. When individuals clicked on the web-link they were brought to the research plain language statement (PLS), followed by the questionnaire.

\section{Procedure}

The respective subscales of the BADS and the standardised AAC-Q and QOLS were combined with the demographic questions to form an online survey presented to participants on the online "Qualtrics" platform. Participants were informed in the PLS that participation was voluntary and that by completing and submitting the questionnaire, they were giving informed consent for their data to be used as part of the study.

Ninety-four individuals completed the questionnaire who met the inclusion criteria. Individuals who did not meet the inclusion criteria (eg., who were not at least 18 years of age) were directed to the end of the questionnaire and informed that they did not meet the requirements necessary to participate.

\section{Data Analysis}

Scores on the AAC-Q and the QOLS were totalled to represent overall total scores of motor coordination function and QOL, respectively. Similarly, scores from the items of the BADS subscales were individually totalled to represent overall scores of avoidance behaviors in relation to their respective areas of investigation.

When participant AAC-Q scores were totalled, 72 scored above the probable DCD cut-off point, 13 scored within the borderline DCD range, while 9 scored below the DCD borderline cut-off point. In order to obtain a larger sample size, the decision was made to initially include individuals who scored within the borderline DCD range, while the 9 participants who scored below the borderline cut-off were excluded from the study, reducing the sample size from 94 to 85 . The same analysis were then run with only those $(\mathrm{N}=72)$ scoring above the borderline cut-off.

All statistical calculations were conducted using the Statistical Package for Social Sciences for Windows (version 21; SPSS Inc., Chicago, IL, USA) with a type 1 error rate of 0.05 . Reliability checks were conducted on each of the scales and subscales used in the study. Descriptive analyses were run to establish mean and standard deviation scores across variables (see Table 1). Several t-tests were run to examine the differences between several demographic groups on scores of QOL. A 
Table 1 Demographic and clinical characteristics of participants with DCD

\begin{tabular}{llll}
\hline Characteristics & Mean & SD & Range \\
\hline Age & 33.14 & 12.05 & $18-63$ \\
Quality of Life (QOLS) & 75.6 & 13.3 & $33-101$ \\
Avoidance & 20.04 & 9.93 & $1-48$ \\
Work/School Impairment & 14.55 & 7.1 & $0-28$ \\
Social Impairment & 9.75 & 6.94 & $0-30$ \\
Motor Coordination (AAC-Q) & 40.6 & 8.75 & $25-60$ \\
& & $\mathrm{~N}$ & $\%$ \\
Sex & & 27 & 31.8 \\
Male & & 58 & 68.2 \\
Female & & & \\
DCD Diagnosis & & 62 & 72.9 \\
Yes & & 23 & 27.1 \\
No & & 23 & \\
DCD Belief & & & \\
Yes & & 27.1 \\
Comorbidity & & 62 & 72.9 \\
Yes & & \\
No & & & \\
\hline
\end{tabular}

Hierarchal Multiple Regression was conducted to access the relationships between the predictor variables (BADS subscales and motor coordination) and QOL.

\section{Results}

Descriptive analyses (See Table 1) revealed that the mean age of the 85 participants (27 males and 58 females) was 33.14 years $(\mathrm{SD}=12.05)$. Sixty-two $(72.9 \%)$ of the participants reported a diagnosis of DCD, while the remaining $23(27.1 \%)$ reported a belief that if clinically assessed, they would receive a DCD diagnosis. Similarly, 23 (27.1\%) participants also reported a diagnosis of a comorbid disorder. The t-test results revealed no significant differences in QOL scores between males and females, or between individuals who did or did not report a comorbid diagnosis.

A Hierarchical Multiple Regression was used to assess the ability of three behavioral avoidance measures (Avoidance, Work/School Impairment and Social Impairment) and motor coordination (AAC-Q Scale) to predict levels of QOL. Prior to conducting a Hierarchical Multiple Regression, preliminary analyses were conducted to test the relevant assumptions. A sample size of 85 was deemed sufficient given that there were four independent variables to be assessed within the analysis. The data also satisfied the assumptions of normality, linearity, multicollinearity, homoscedasticity and independence of residuals.

A four stage hierarchical multiple regression was carried out with QOL as the outcome variable. The three behavioral avoidance scales were entered as the first three blocks, with the Avoidance entered as step 1, Work/School Impairment as Step 2, Social Impairment as Step 3 and Motor Coordination (AAC-Q scale) as Step 4. 
Despite the fact that previous literature suggests that motor coordination is related to QOL within the DCD population, the three behavioral avoidance subscales were all entered prior the inclusion of motor coordination. The decision was made to prioritise the exploration of the concept of behavioral avoidance within the DCD population.

Entered as Step 1, the Avoidance scale explained 18.5\% (See Fig. 1) of the variance in QOL, $F(1,83)=18.9, p<0.001$. The inclusion of Work/School Impairment as Step 2 resulted in the model now accounting for $25.9 \%$ of the variance in QOL. Work/School Impairment explained an additional 7.3\% of variation in QOL and this change in $\mathrm{R}^{2}$ was significant, $\mathrm{F}(1,82)=8.08, \mathrm{p}<0.006$. Following the addition of Social Impairment, the model accounted for $34.5 \%$ of the variance in QOL with Social Impairment explaining an additional $8.6 \%$ of this variance. This change in $\mathrm{R}^{2}$ was also significant, $\mathrm{F}(1,81)=10.68, \mathrm{p}<0.002$. Finally, the inclusion of Motor Coordination did not significantly add to the model of QOL, F $(1,80)=0.405$, $\mathrm{p}>0.05$. Together, the four independent variables accounted for $34.8 \%$ of the variance in QOL. In the final model, only two of the predictors were deemed statistically significant (See Table 2), with Social Impairment recording a higher beta value (beta $=-0.386, p<0.005)$ than Work/School Impairment (beta $=-0.23, p<0.05$ ).

For comparative purposes, the same steps were followed to run a hierarchical regression with only those participants $(\mathrm{N}=72)$ who score above the borderline cutoff. In this second analysis the Avoidance scale, when entered at Step 1, explained $12.9 \%$ (See Fig. 2) of the variance in QOL, $F(1,70)=10.38, p<0.01$. The inclusion of Work/School Impairment as Step 2 explained an additional $7.1 \%$ of variation in QOL and this change in $\mathrm{R}^{2}$ was significant, $\mathrm{F}(1,69)=6.15, \mathrm{p}<0.05$. This resulted in the model now accounting for $20 \%$ of the variance in QOL. With the addition of

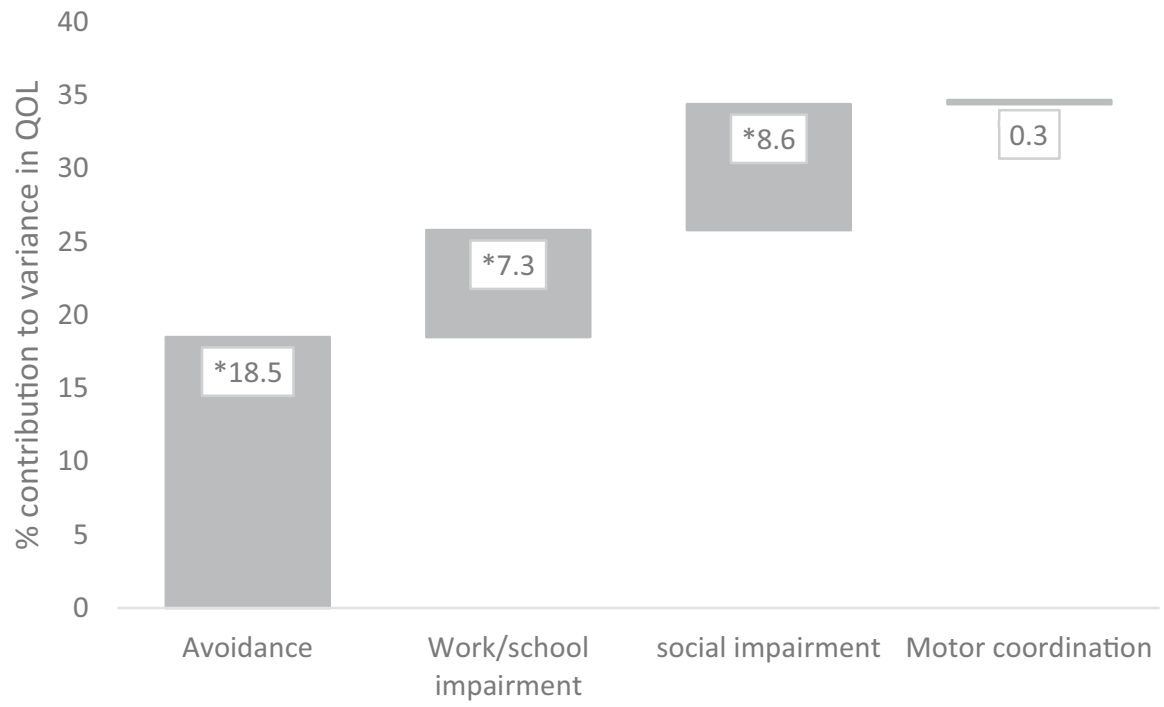

Fig. 1 The relative contribution of each step in the first hierarchical regression $(\mathrm{N}=85)$ to the variance in QOL. Significant associations are indicated by * 


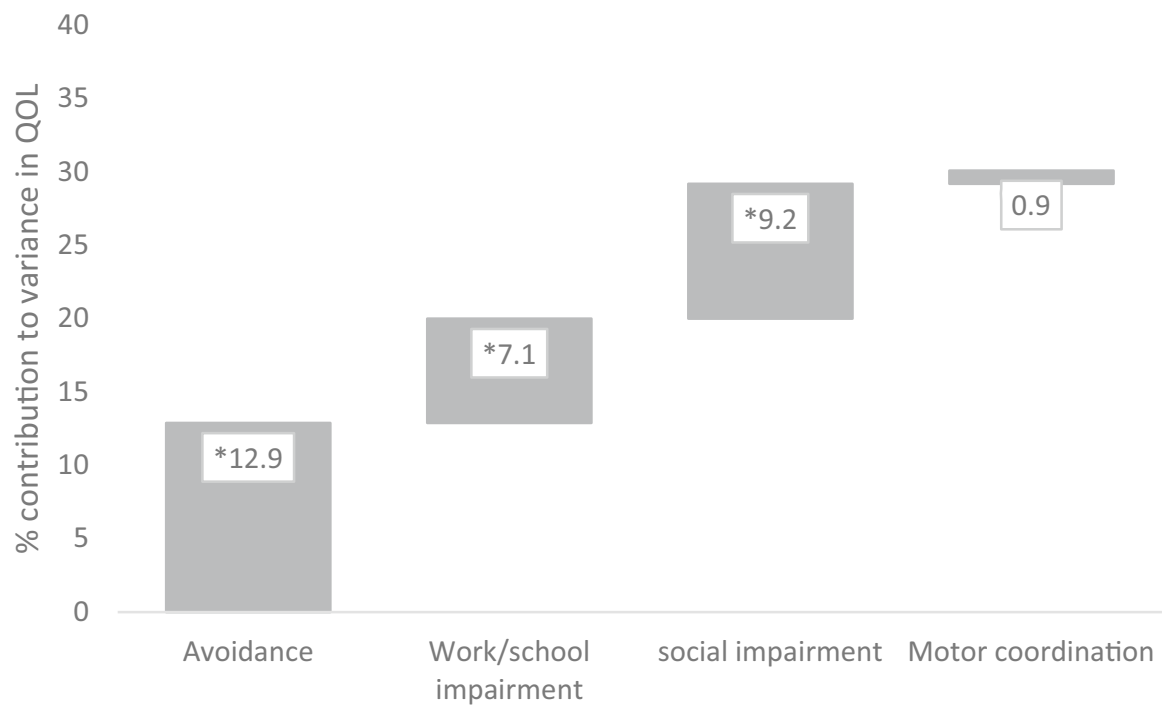

Fig. 2 The relative contribution of each step in the second hierarchical regression including participants above the borderline cut-off for the AAC-Q $(\mathrm{N}=72)$ to the variance in QOL. Significant associations are indicated by $*$

Social Impairment, the model accounted for $29.2 \%$ of the variance in QOL. Social Impairment explained an additional $9.2 \%$ of this variance and this change in $\mathrm{R}^{2}$ was also significant, $\mathrm{F}(1,68)=8.80, \mathrm{p}<0.01$. As before, the inclusion of Motor Coordination did not significantly add to the model of QOL, F $(1,67)=0.887, \mathrm{p}>0.05$. Together, the four independent variables accounted for $34.7 \%$ of the variance in QOL. In the final model, the only predictor deemed statistically significant (See Table 3), was Social Impairment (beta $=-0.389, \mathrm{p}<0.005$ ).

\section{Discussion}

DCD was previously considered to be a childhood disorder. Although more is now known about it in adulthood, there remain considerable gaps in our knowledge of the wellbeing of adults with a diagnosis, or suspected diagnosis, of DCD. The purpose of the current research was therefore to conduct the first known study measuring avoidance behaviours in an adult DCD population and the impact of these on QOL. Adults with DCD were assessed on self-reported levels of motor coordination, engagement in avoidance behaviors and QOL. The study revealed that the three subscales of avoidance behavior (Avoidance, Work/School Impairment and Social Impairment) assessed were each significantly correlated with scores of QOL. More specifically, as hypothesised, higher scores in each measure of avoidance behavior was associated with lower scores on QOL. The mean scores on each individual avoidance behavior subscale reported by the participants in this study were also much higher than the mean scores recorded by Kanter et al. (2007) in a random 


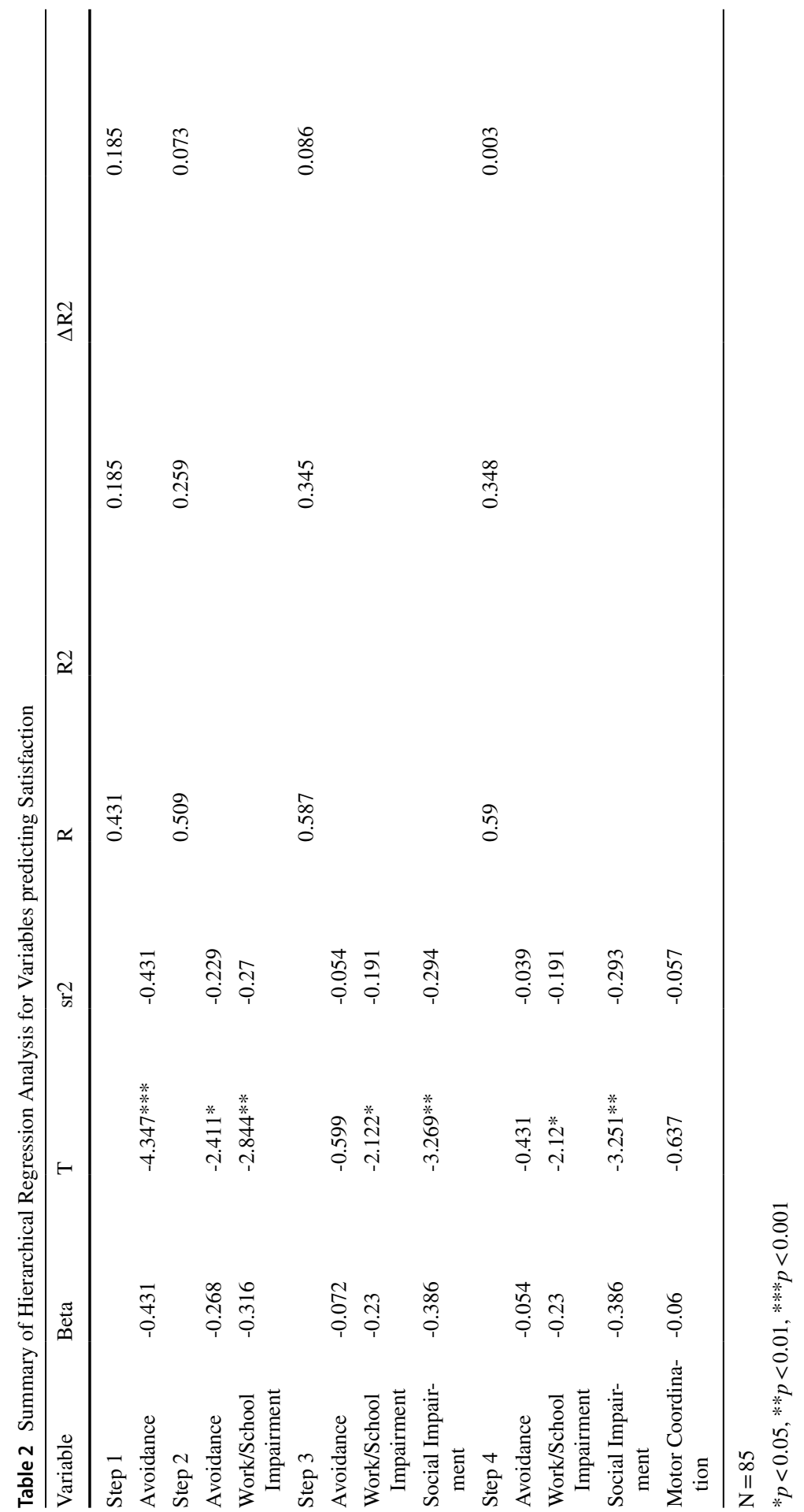




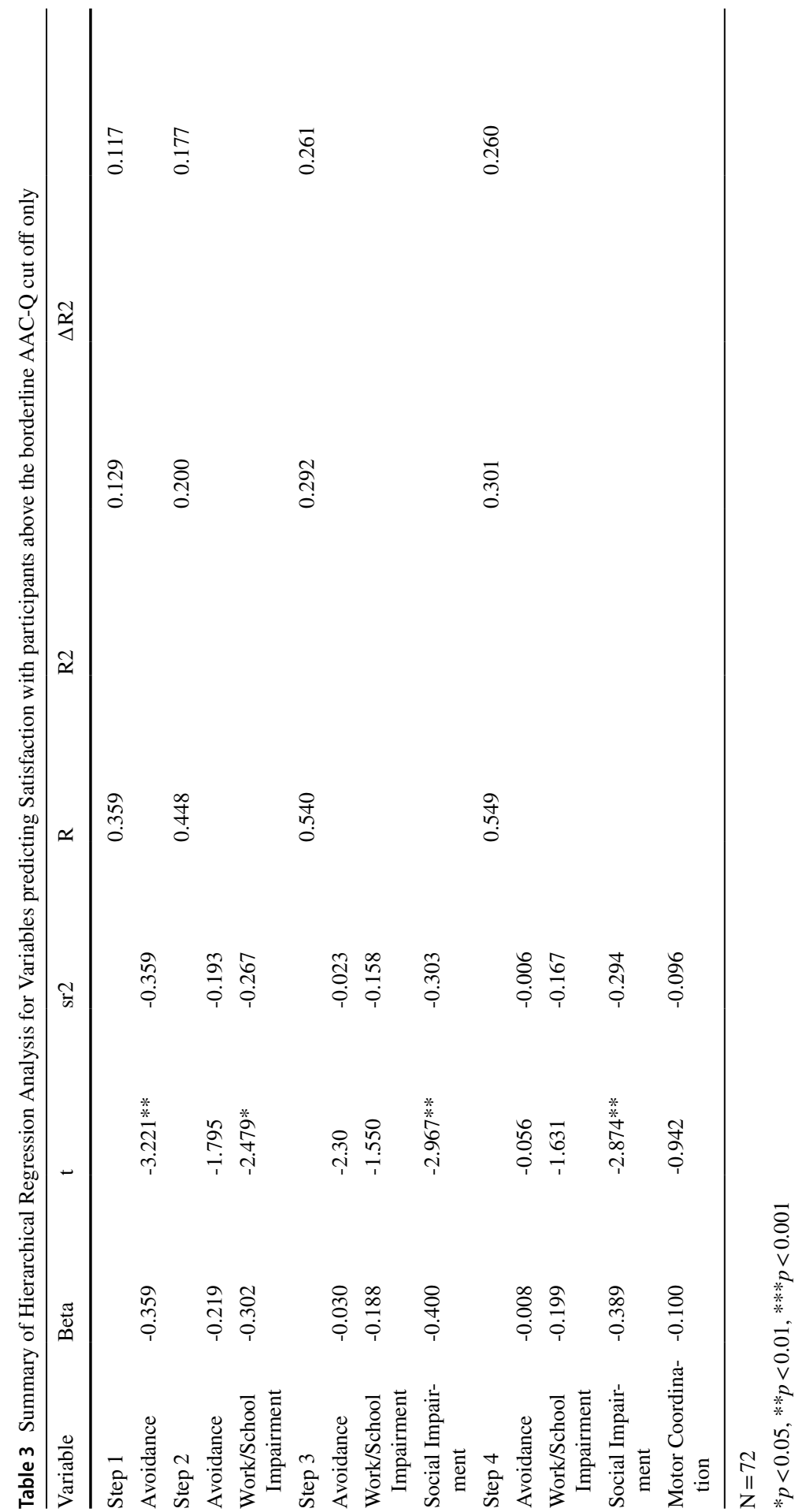


sample of 319 adult participants. Although it was predicted that higher levels of motor impairment would be significantly associated with lower scores on QOL, this was not found to be the case. The findings from the current study are the first to provide evidence to suggest that adults with DCD engage in more avoidance behavior than their neurotypical counterparts and that such avoidance behaviors are directly associated with QOL.

A substantial body of literature has assessed levels of participation within a DCD population, suggesting that individuals with DCD engage significantly less in participation than their non-DCD counterparts (Cousins \& Smyth, 2003; Kirby et al., 2011b; Mandich et al., 2003; Poulsen et al., 2007; Rosenblum \& Engel-Yeger, 2014; Sylvestre et al., 2013). However, no known research has focused specifically on avoidance behaviours which are quite different from non-participation. The current research therefore provides new insights into the experiences of adults to DCD. Along with suggesting that adults with DCD actively avoid certain situations, the results also suggest that the motivation for non-participation may be that participation in areas such as school, work and in social situations may not be a priority of adults with DCD. As such, this may partially explain why previous studies found significantly lower participation levels in the DCD population.

It was hypothesised higher scores on three subscales of the BADS would be associated with poorer QOL scores and this was indeed found to be the case. Slight differences in the contribution of each subscale to the variance in quality of life were found depending on whether or not participants below the borderline cut-off on the AAC-Q were included in the analysis. This is interesting and highlights the careful consideration needed when including borderline participants in analysis although the authors of the AAC-Q suggest that they can be grouped together with those above the borderline cut-off. The mean BADS subscale scores recorded in the current study were all higher that reported in a general population. Within a random sample of 319 undergraduate students, Kanter et al. (2007) reported mean scores of 14.16 for Avoidance, 8.08 for Work/School Impairment and 3.42 for Social Impairment. By contrast, in the current sample, mean scores of 20.04, 14.55 and 9.75 were reported for each subscale respectively.

Taking Avoidance as the first, BADS subscale, both analysis including and excluding participants below the borderline cut-off on the AAC-Q suggested that this was the subscale most strongly correlated to scores of QOL. As aforementioned, scores on the "Avoidance" subscale represents avoidance of negative aversive states and engaging in rumination rather than active problem solving. Thus, the strong relationship observed in this study suggests that individuals with DCD who report higher levels of actively avoiding negative aversive states and engagement in more rumination, are more likely to report poorer QOL. The "Avoidance" mean score of 20.04 for the overall sample was also substantially greater than the mean score of 14.16 reported in a random sample of 319 college students (Kanter et al., 2007), suggesting that adults with DCD engage in more avoidance and rumination behavior than typically developed adults. The results also provide support for previous literature, which suggests that young adults with DCD tend to demonstrate low levels of participation in potentially aversive physical activities such as team sports, driving and dancing (Cousins \& Smyth, 2003; Kirby et al., 2011a) but also in social, 
educational and employment situations (Kirby et al., 2011a, 2011b; Tal-Saban et al., 2014a, 2014b). The current research suggestions that this may go beyond simple non participation to active avoidance of many activities and aspects of life. This provides a first step into gaining much needed insight into function of non-participation. Further research on the potential avoidance involved is needed to elucidate this and properly understand the function of behaviour, or non-behaviour, in these contexts.

Higher scores of the second BADS subscale, "Work/School Impairment", were also found to be associated with lower scores of QOL within both the complete DCD adult sample, and those above the borderline only. This suggests that the consequences of inactivity and passivity on work and school responsibilities negatively impacts QOL within this population. This is not surprising given that studies of adults with DCD within educational and work settings indicate that they experience motor-related difficulties such as handwriting and executive functioning deficits which makes it difficult to meet the demands of school and work (Barnett et al., 2011; Kirby et al., 2008). Kirby et al. (2013) report that adults with DCD often find it difficult to retain employment status. Studies of children with DCD in school have suggested that they prefer to spend more time alone (Smyth \& Anderson, 2000). The current study provides evidence to suggest that such avoidant behavior exhibited during childhood in school may persist into adulthood, negatively affecting their education, work responsibilities and QOL.

The items of the third BADS subscale, "Social Impairment", represent a measure of social isolation and the consequences of social inactivity and passivity (Kanter et al., 2007). Higher scores on this subscale were found to be significantly correlated with lower scores of QOL. Indeed, in the second regression performed without participants below the borderline cut-off on the AAC-Q, this was the subscale of the BADS second most strongly correlated with QOL scores after Avoidance. When all participants were included it was the subscale of the BADS demonstrating the weakest (although still significant correlation with QOL). This provides support for previous research which has suggested that social withdrawal and isolation may partially explain why individuals with DCD report poorer QOL than their neurotypical peers (Cousins \& Smyth, 2003; Kirby et al., 2011b; Mandich et al., 2003; Tal-Saban et al., 2014a). It also opens the door for future research to explore the relationship between degree of motor impairment and social impairment and avoidance in DCD. Our second hypothesis that motor impairment would be significantly associated with poorer QOL of life was based upon existing findings. Tal-Saban et al. (2014a) compared QOL scores between individuals with DCD or borderline DCD and individuals who had no suspected DCD, with the results indicating that adolescents and adults with DCD or borderline DCD have poorer QOL than their typically developed counterparts. The researchers suggest that this difference may be due to the motor coordination difficulties experienced by individuals with DCD. In order to investigate this further, the current study measured motor coordination and assessed its relationship with QOL within individuals within DCD or borderline DCD groups only. Our results did not suggest that levels of motor coordination directly influences QOL within this particular DCD population. Critically, in combination with our other findings, this serves to highlight the relative importance of factors other than motor coordination on wellbeing and QOL in an adult DCD population. Although 
our results did not support the claims of Tal-Saban et al. (2014a), the fact that the removal of the borderline cases did not significantly change this finding is of note. This lends weight to the suggestion by authors of the AAC-Q that borderline cases can be meaningfully included in analyses. However, the relatively high percentage of female relative to male participants is at odds with reported prevalence rates and may contribute to the discrepant findings. Given that females typically report impairment in gross motor and non-motor difficulties while males report challenges with fine motor difficulties (Cleaton et al., 2021). It is therefore possible that the females sampled presented primarily with non-motor rather than motor difficulties.

A number of limitations should be noted when interpreting the current findings. One issue of particular note that is more objective, physical measures of motor coordination may have advantages over self-reports, and future research should look to incorporate these in order to strengthen the participant inclusion and screening criteria. Additionally as previously highlighted, although the prevalence of DCD being much greater in males, the participants of the current study consisted of only 27 males $(31.8 \%)$, in comparison to 58 females $(68.2 \%)$. Therefore, the sample may not accurately represent the DCD adult population and thus reduces the ecological validity of the results. This imbalance may be partially explained by research suggesting that females may be more willing to participate in social research (Markanday et al., 2013). Similarly, the inclusion of 12 participants who scored within the borderline DCD range on the AAC-Q may also limit the generalisability of this study. This is despite Tal-Saban et al. (2014b) indicating that although it is not preferable, it is acceptable to include individuals who score within this range in DCD research. As the study took the form of an online questionnaire, it also meant that individuals with severe DCD symptoms, both motor and non-motor, may not have participated due to the coordination skills required to complete the questionnaire. Although less convenient, future research of a similar nature should consider using phone calls and face to face meetings as forms of data collection in order to obtain a more representative DCD sample.

Despite some limitations, this study has important implications for future DCD research and for the QOL of adults with DCD. It is the first study of its kind that has specifically examined levels of avoidance behavior within the DCD adult population and its relationship with QOL. Given that the results yielded indicate that avoidance behavior significant influences QOL, it is important that future research attempts to replicate and expand on these findings. It is also important that future research attempts to establish successful interventions which can help reduce avoidance behavior and ultimately increase the QOL of adults with DCD. Behavior Activation (BA) treatment has emerged as a cost effective form of intervention for reducing depression (Porter et al., 2004) and Post-Traumatic Stress Disorder (PTSD) symptoms in veterans (Jakupcak et al., 2006). Future researchers may wish to explore whether the underlying concepts of BA treatment can be used as the foundation upon which an effective intervention treatment for adults with DCD can be based, whereby it reduces engagement in avoidance behaviors, improves behavioral activation and ultimately leads to improved QOL.

To conclude, our study suggests that adults with DCD actively avoid potentially aversive social situations in everyday life, including in school and workplace 
settings. The findings also suggest that those who engage in more avoidance behaviors are more likely to experience lower QOL than those who engage less frequently in active avoidance. However, the results did not provide evidence to suggest that motor coordination has a direct impact on QOL. Overall, the findings from this study highlight the need for further research exploration of the concept of avoidance behavior within the DCD adult population in order to develop effective intervention treatments which can be utilised by clinicians to improve the QOL of adults with DCD.

Author Contributions This study was conducted by the first author under the supervision of the second author. Both authors contributed to the study conception and design. Material preparation, data collection were performed by the first author. Analysis was conducted by both authors. The first draft of the manuscript was written by the first author and both authors commented on previous versions of the manuscript. Both authors read and approved the final manuscript.

Funding Open Access funding provided by the IReL Consortium.

Availability of Data and Material Informed consent was not sought to share data in an open format.

\section{Declarations}

Ethical Approval was obtained from the Psychology Ethics Committee at Dublin City University and the study was performed in accordance with the ethical standards as laid down in the 1964 Declaration of Helsinki and its later amendments..

Conflict of Interest The authors declare no conflict of interests.

Consent to Participate All participants gave informed consent to take part in the study.

Consent for Publication All authors consent to the publication of this work.

Open Access This article is licensed under a Creative Commons Attribution 4.0 International License, which permits use, sharing, adaptation, distribution and reproduction in any medium or format, as long as you give appropriate credit to the original author(s) and the source, provide a link to the Creative Commons licence, and indicate if changes were made. The images or other third party material in this article are included in the article's Creative Commons licence, unless indicated otherwise in a credit line to the material. If material is not included in the article's Creative Commons licence and your intended use is not permitted by statutory regulation or exceeds the permitted use, you will need to obtain permission directly from the copyright holder. To view a copy of this licence, visit http://creativecommons.org/ licenses/by/4.0/.

\section{References}

Barnett, A. L., Henderson, S. E., Scheib, B., \& Schulz, J. (2011). Handwriting difficulties and their assessment in young adults with DCD: Extension of the DASH for 17-to 25-year-olds. Journal of Adult Development, 18(3), 114-121. https://doi.org/10.1007/s10804-011-9121-3

Blank, R., Smits-Engelsman, B., Polatajko, H., \& Wilson, P. (2012). European Academy for Childhood Disability (EACD): Recommendations on the definition, diagnosis and intervention of developmental coordination disorder (long version). Developmental Medicine \& Child Neurology, 54(1), 54-93. https://doi.org/10.1111/j.1469-8749,2011.04171 
Borge, C. R., Wahl, A. K., \& Moum, T. (2011). Pain and quality of life with chronic obstructive pulmonary disease. Heart \& Lung: THe Journal of Acute and Critical Care, 40(3), e90-e101. https://doi. org/10.1016/j.hrtlng.2010.10.009

Burckhardt, C. S., \& Anderson, K. L. (2003). The Quality of Life Scale (QOLS): Reliability, validity, and utilization. Health and Quality of Life Outcomes, 1(1), 60. https://doi.org/10.1186/1477-7525-1-60

Burckhardt, C. S., Woods, S. L., Schultz, A. A., \& Ziebarth, D. M. (1989). Quality of life of adults with chronic illness: A psychometric study. Research in Nursing \& Health, 12(6), 347-354.

Cantell, M. H., Smyth, M. M., \& Ahonen, T. P. (2003). Two distinct pathways for developmental coordination disorder: Persistence and resolution. Human Movement Science, 22(4), 413-431. https://doi. org/10.1016/j.humov.2003.09.002

Cantell, M. H., Smyth, M. M., \& Ahonen, T. P. (1994). Clumsiness in adolescence: Educational, motor, and social outcomes of motor delay detected at 5 years. Adapted Physical Activity Quarterly, 11(2), 115-129. https://doi.org/10.1123/apaq.11.2.115

Cleaton, M. A., Tal-Saban, M., Hill, E. L., \& Kirby, A. (2021). Gender and age differences in the presentation of at-risk or probable Developmental Coordination Disorder in adults. Research in Developmental Disabilities, 115, 104010. https://doi.org/10.1016/j.ridd.2021.104010

Cousins, M., \& Smyth, M. M. (2003). Developmental coordination impairments in adulthood. Human Movement Science, 22(4), 433-459. https://doi.org/10.1016/j.humov.2003.09.003

de Oliveira, R. F., \& Wann, J. P. (2011). Driving skills of young adults with developmental coordination disorder: Regulating speed and coping with distraction. Research in Developmental Disabilities, 32(4), 1301-1308. https://doi.org/10.1016/j.ridd.2010.12.021

Dettling-Ihnenfeldt, D. S., de Graaff, A. E., Beelen, A., Nollet, F., \& van der Schaaf, M. (2016). Coping style and quality of life in Dutch intensive care unit survivors. Rehabilitation Psychology, 61(2), 165. https://doi.org/10.1037/rep0000084

Dewey, D., Kaplan, B. J., Crawford, S. G., \& Wilson, B. N. (2002). Developmental coordination disorder: Associated problems in attention, learning, and psychosocial adjustment. Human Movement Science, 21(5), 905-918. https://doi.org/10.1016/S0167-9457(02)00163-X

Flapper, B. C., \& Schoemaker, M. M. (2013). Developmental coordination disorder in children with specific language impairment: Co-morbidity and impact on quality of life. Research in Developmental Disabilities, 34(2), 756-763. https://doi.org/10.1016/j.ridd.2012.10.014

Geuze, R., \& Börger, H. (1993). Children who are clumsy: Five years later. Adapted Physical Activity Quarterly, 10(1), 10-21. https://doi.org/10.1123/apaq.10.1.10

Gillberg, I. C., \& Gillberg, C. (1989). Children with preschool minor neurodevelopmental disorders. IV: Behaviour and school achievement at age 13. Developmental Medicine \& Child Neurology, 31(1), 3-13. https://doi.org/10.1111/j.1469-8749.1989.tb08406.x

Hill, E. L., \& Brown, D. (2013). Mood impairments in adults previously diagnosed with developmental coordination disorder. Journal of Mental Health, 22(4), 334-340. https://doi.org/10.3109/09638237. 2012.745187

Hill, E. L., Brown, D., \& Sorgardt, K. S. (2011). A preliminary investigation of quality of life satisfaction reports in emerging adults with and without developmental coordination disorder. Journal of Adult Development, 18(3), 130-134. https://doi.org/10.1007/s10804-011-9122-2

Jakupcak, M., Roberts, L. J., Martell, C., Mulick, P., Michael, S., Reed, R., \& McFall, M. (2006). A pilot study of behavioral activation for veterans with posttraumatic stress disorder. Journal of Traumatic Stress, 19(3), 387-391. https://doi.org/10.1002/jts.20125

Kanter, J. W., Mulick, P. S., Busch, A. M., Berlin, K. S., \& Martell, C. R. (2007). The Behavioural Activation for Depression Scale (BADS): Psychometric properties and factor structure. Journal of Psychopathology and Behavioural Assessment, 29(3), 191-202. https://doi.org/10.1007/ s10862-006-9038-5

Kaplan, B. J., Dewey, D. M., Crawford, S. G., \& Wilson, B. N. (2001). The term comorbidity is of questionable value in reference to developmental disorders data and theory. Journal of Learning Disabilities, 34(6), 555-565. https://doi.org/10.1177/002221940103400608

Kirby, A., Williams, N., Thomas, M., \& Hill, E. L. (2013). Self-reported mood, general health, wellbeing and employment status in adults with suspected DCD. Research in Developmental Disabilities, 34(4), 1357-1364. https://doi.org/10.1016/j.ridd.2013.01.003

Kirby, A., Sugden, D., \& Edwards, L. (2011a). Driving behaviour in young adults with developmental co-ordination disorder. Journal of Adult Development, 18(3), 122-129. https://doi.org/10.1007/ s10804-011-9120-4 
Kirby, A., Edwards, L., \& Sugden, D. (2011b). Emerging adulthood and developmental co-ordination disorder. Journal of Adult Development, 18(3), 107-113. https://doi.org/10.1007/s10804-011-9123-1

Kirby, A., Sugden, D., Beveridge, S., \& Edwards, L. (2008). Developmental co-ordination disorder (DCD) in adolescents and adults in further and higher education. Journal of Research in Special Educational Needs, 8(3), 120-131. https://doi.org/10.1111/j.1471-3802.2008.00111.x

Liberman, L., Ratzon, N., \& Bart, O. (2013). The profile of performance skills and emotional factors in the context of participation among young children with Developmental Coordination Disorder. Research in Developmental Disabilities, 34(1), 87-94. https://doi.org/10.1016/j.ridd.2012.07.019

Losse, A., Henderson, S. E., Elliman, D., Hall, D., Knight, E., \& Jongmans, M. (1991). Clumsiness in children-do they grow out of it? A 10-year follow-up study. Developmental Medicine \& Child Neurology, 33(1), 55-68. https://doi.org/10.1111/j.1469-8749.1991.tb14785.x

Mandich, A. D., Polatajko, H. J., \& Rodger, S. (2003). Rites of passage: Understanding participation of children with developmental coordination disorder. Human Movement Science, 22(4), 583-595. https://doi.org/10.1016/j.humov.2003.09.011

Markanday, S., Brennan, S. L., Gould, H., \& Pasco, J. A. (2013). Sex-differences in reasons for nonparticipation at recruitment: Geelong Osteoporosis Study. BMC Research Notes, 6(1), 104. https:// doi.org/10.1186/1756-0500-6-104

Megari, K. (2013). Quality of Life in Chronic Disease Patients. Health Psychology Research, 1(3), e27. https://doi.org/10.4081/hpr.2013.e27

Missiuna, C., Gaines, R., \& Soucie, H. (2006). Why every office needs a tennis ball: A new approach to assessing the clumsy child. Canadian Medical Association Journal, 175(5), 471-471. https://doi. org/10.1503/cmaj.051202

Ottenbreit, N. D., \& Dobson, K. S. (2004). Avoidance and depression: The construction of the CognitiveBehavioral Avoidance Scale. Behaviour Research and Therapy, 42(3), 293-313. https://doi.org/10. 1016/S0005-7967(03)00140-2

Patrick, D. L., Kinne, S., Engelberg, R. A., \& Pearlman, R. A. (2000). Functional status and perceived quality of life in adults with and without chronic conditions. Journal of Clinical Epidemiology, 53(8), 779-785. https://doi.org/10.1016/S0895-4356(00)00205-5

Petts, R. A., Foster, C. S., Douleh, T. N., \& Gaynor, S. T. (2016). Measuring activation in adolescent depression: Preliminary psychometric data on the Behavioral Activation for Depression Scale-Short Form. Behavior Analysis: Research and Practice, 16(2), 65. https://doi.org/10.1037/bar0000036

Piek, J. P., Dworcan, M., Barrett, N. C., \& Coleman, R. (2000). Determinants of self-worth in children with and without developmental coordination disorder. International Journal of Disability, Development and Education, 47(3), 259-272. https://doi.org/10.1080/713671115

Porter, J. F., Spates, C. R., \& Smitham, S. (2004). Behavioral activation group therapy in public mental health settings: A pilot investigation. Professional Psychology: Research and Practice, 35(3), 297. https://doi.org/10.1037/0735-7028.35.3.297

Poulsen, A. A., Ziviani, J. M., Cuskelly, M., \& Smith, R. (2007). Boys with developmental coordination disorder: Loneliness and team sports participation. American Journal of Occupational Therapy, 61(4), 451-462. https://doi.org/10.5014/ajot.61.4.451

Rosenblum, S., \& Engel-Yeger, B. (2014). Predicting participation in Children with DCD. Current Developmental Disorders Reports, 1(2), 109-117. https://doi.org/10.1007/s40474-014-0014-6

Schoemaker, M. M., \& Kalverboer, A. F. (1994). Social and affective problems of children who are clumsy: How early do they begin? Adapted Physical Activity Quarterly, 11(2), 130-140. https://doi. org/10.1123/apaq.11.2.130

Shaw, L., Levine, M. D., \& Belfer, M. (1982). Developmental double jeopardy: A study of clumsiness and self-esteem in children with learning problems. Journal of Developmental \& Behavioral Pediatrics, 3(4), 191-196. https://doi.org/10.1097/00004703-198212000-00003

Smyth, M. M., \& Anderson, H. I. (2000). Coping with clumsiness in the school playground: Social and physical play in children with coordination impairments. British Journal of Developmental Psychology, 18(3), 389-413. https://doi.org/10.1348/026151000165760

Sylvestre, A., Nadeau, L., Charron, L., Larose, N., \& Lepage, C. (2013). Social participation by children with developmental coordination disorder compared to their peers. Disability and Rehabilitation, 35(21), 1814-1820. https://doi.org/10.3109/09638288.2012.756943

Tal-Saban, M., Ornoy, A., \& Parush, S. (2014a). Young adults with developmental coordination disorder: A longitudinal study. American Journal of Occupational Therapy, 68(3), 307-316. https://doi.org/ 10.5014/ajot.2014.009563 
Tal-Saban, M., Ornoy, A., \& Parush, S. (2014b). Executive function and attention in young adults with and without Developmental Coordination Disorder-A comparative study. Research in Developmental Disabilities, 35(11), 2644-2650. https://doi.org/10.1016/j.ridd.2014.07.002

Tal-Saban, M., Zarka, S., Grotto, I., Ornoy, A., \& Parush, S. (2012a). The functional profile of young adults with suspected Developmental Coordination Disorder (DCD). Research in Developmental Disabilities, 33(6), 2193-2202. https://doi.org/10.1016/j.ridd.2012.06.005

Tal-Saban, M., Ornoy, A., Grotto, I., \& Parush, S. (2012b). Adolescents and adults coordination questionnaire: Development and psychometric properties. American Journal of Occupational Therapy, 66(4), 406-413. https://doi.org/10.5014/ajot.2012.003251

Van Campen, C., \& Iedema, J. (2007). Are persons with physical disabilities who participate in society healthier and happier? Structural equation modelling of objective participation and subjective wellbeing. Quality of Life Research, 16(4), 635. https://doi.org/10.1007/s11136-006-9147-3

Wahl, A., Burckhardt, C., Wiklund, I., \& Hanestad, B. R. (1998). The Norwegian Version of the Quality of Life Scale (QOLS-N) A Validation and Reliability Study in Patients Suffering from Psoriasis. Scandinavian Journal of Caring Sciences, 12(4), 215-222

Wahl, A. K., Rustøen, T., Hanestad, B. R., Lerdal, A., \& Moum, T. (2004). Quality of life in the general Norwegian population, measured by the Quality of Life Scale (QOLS-N). Quality of Life Research, 13(5), 1001-1009.

WHOQOL Group. (1995). The World Health Organization quality of life assessment (WHOQOL): Position paper from the World Health Organization. Social Science \& Medicine, 41(10), 1403-1409. https://doi.org/10.1016/0277-9536(95)00112-K

Zeiss, A. M., Lewinsohn, P. M., \& Muñoz, R. F. (1979). Nonspecific improvement effects in depression using interpersonal skills training, pleasant activity schedules, or cognitive training. Journal of Consulting and Clinical Psychology, 47(3), 427. https://doi.org/10.1037/0022-006X.47.3.427

Zwicker, J. G., Harris, S. R., \& Klassen, A. F. (2013). Quality of life domains affected in children with developmental coordination disorder: a systematic review. Child: Care, Health and Development, 39(4), 562-580. https://doi.org/10.1111/j.1365-2214.2012.01379.x

Publisher's Note Springer Nature remains neutral with regard to jurisdictional claims in published maps and institutional affiliations. 